\title{
Isolation of colistin-resistant Hafnia alvei
}

Colistin belongs to the polymyxins, a group of polypeptide antibiotics which includes polymyxins A, B, C, D and colistin (polymyxin E). Of these, only polymyxins $\mathrm{B}$ and $\mathrm{E}$ have been employed for therapy of human infections (Gales et al., 2001; Kasiakou et al., 2005; Tan \& $\mathrm{Ng}, 2006)$. Colistin is mainly administered as colistin sulphomethate sodium; the active drug is then released after hydrolysis and removed by glomerular filtration (Muyembe et al., 1973). Bactericidal activity is due to binding of cell membrane phospholipids and subsequent rapid permeability changes, leading to leakage of cell contents. Interestingly, this process is not dependent on bacterial metabolic activity (Gales et al., 2001; Kasiakou et al., 2005; Tan \& Ng, 2006). Colistin emerged in the early 1960s (Catchpole et al., 1997; Kasiakou et al., 2005) as an alternative for treatment of multidrug-resistant Klebsiella pneumoniae, Acinetobacter baumannii and Pseudomonas aeruginosa infections, but due to its neurotoxicity (neuromuscular blockade, dizziness, nausea, convulsions, coma) and nephrotoxicity (Gales et al., 2001; Jones et al., 2005; Kasiakou et al., 2005; Tan \& Ng, 2006) it was displaced in the 1970s by the less toxic aminoglycosides, carboxypenicillins and cephalosporins. Clinical use of colistin has therefore been limited to use in oral nonabsorbable compounds, nebulized formulations, and topical preparations for therapy of otitis, conjunctivitis and skin infections (Jones et al., 2005; Tan \& Ng, 2006). For this reason, our knowledge of colistin is limited. It exerts activity against Gram-negative bacteria [including extended-spectrum $\beta$-lactamase (ESBL)producing bacteria] (Catchpole et al., 1997), except for Proteus spp., Serratia spp., Providencia spp., Burkholderia pseudomallei and Neisseria spp., which are known to be intrinsically resistant to colistin. Also, Gram-positive and anaerobic organisms show intrinsic polymyxin resistance (Kasiakou et al., 2005; Li et al., 2005; Markou et al., 2003). In addition, resistant $P$. aeruginosa,
Acinetobacter, Edwardsiella tarda, Enterobacter, Klebsiella and Salmonella strains have been reported (Catchpole et al., 1997; Kasiakou et al., 2005; Muyembe et al., 1973; Shimizu et al., 1977). Interestingly, cross-resistance with antimicrobials other than polypeptide compounds has not been described so far. Recently, the paucity of novel antibiotics against multidrugresistant organisms has renewed interest in the possible use of polymyxin E.

Three Hafnia alvei strains were isolated from faeces of hospitalized leukaemia patients from January 2004 to June 2007. Identifications were obtained by VITEK 2 (card ID-GNB) and confirmed by the mini API system (both by bioMérieux). Susceptibilities were provided by VITEK 2 (AST-N021, AST-N022 and AST-N041 cards; bioMérieux), and confirmed by performing a disc diffusion test on Mueller-Hinton agar (bioMérieux) (NCCLS, 2003) (see Table 1). Cefoxitin, ampicillin, amoxicillin-clavulanate, cefotaxime, ceftazidime, cefpirome, imipenem, meropenem, amikacin, ciprofloxacin, cotrimoxazole, tetracycline and colistin discs were used (discs from Liofilchem). Plates were incubated for $24 \mathrm{~h}$ at $36{ }^{\circ} \mathrm{C}$ in ambient air. Of particular note, two of the three $H$. alvei isolates were found to show colistin resistance. Also, E-test with cefotaxime plus cefotaxime/ clavulanate and ceftazidime plus ceftazidime/clavulanate (AB BIODISK) was carried out on Mueller-Hinton agar, showing the absence of ESBL production.

\section{$H$. alvei causes uncommon nosocomial} infections including cholecystitis, airway, urinary tract, liver and pancreatic infections, sporadic cases of enteritis, conjunctivitis, joint and wound infections, pleuritis, peritonitis and bacteraemia (Catchpole et al., 1997; Janda \& Abbott, 2006; Jones et al., 2005; Kasiakou et al., 2005; Li et al., 2005). This organism commonly shows susceptibility to quinolones, aminoglycosides, chloramphenicol, cotrimoxazole, cefepime, aztreonam and carbapenems, whereas susceptibility to tetracycline is variable. Penicillin and narrow-spectrum cephalosporin resistance has been reported, and has been found to be due to a natural high-level constitutive chromosomal Bush group $1 \beta$-lactamase (resistant to oxyiminocephalosporins) or to a low-level inducible Bush group 1 cephalosporinase (susceptible to oxyimino-cephalosporins). An uncommon AmpC $\beta$-lactamase may be responsible for ceftazidime and cefotaxime resistance, and for reduced susceptibility to cefpirome (Girlich et al., 2000; Janda \& Abbott, 2006; Thomson et al., 1993). To our knowledge, $H$. alvei colistin resistance has not been described before.

The three patients studied suffered from diarrhoea, while abdominal pain and fever were absent. In all of the three cases $H$. alvei was collected with high bacterial counts ( $>100$ c.f.u. per plate) but as part of the mixed flora. Also, no other organism (such as Shigella spp., Yersinia spp., Salmonella spp., Clostridium difficile) of known enteric pathogenicity was isolated, and C. difficile antigen and A/B toxins were not detected by the immunoenzymic Clostridium difficile Panel test (Biosite). Therefore, the role of $H$. alvei as the agent of the episodes of diarrhoea remained unclear. Also, H. alvei is a member of the family Enterobacteriaceae, but its enteropathogenicity remains controversial at present (Janda \& Abbott, 2006).

Though reports concerning $H$. alvei isolation are still uncommon, emergence of colistin resistance represents a serious clinical and microbiological concern, as an increased incidence of hospital infections caused by multidrug-resistant Gramnegative organisms has been observed over the past years in Italy as well as worldwide, and has justified the reintroduction of colistin as a promising alternative in the treatment of severe life-threatening infections. Particularly, critically ill patients admitted to medical and surgical intensive care units and suffering from pneumonia and bloodstream infections caused by multidrug-resistant but colistin- 
Table 1. MIC values $\left(\mu \mathrm{g} \mathrm{ml}^{-1}\right)$ for the $H$. alvei strains and their interpretation

S, Susceptible; R, resistant; FOX, cefoxitin; AMP, ampicillin; AUG, amoxicillin-clavulanate; CTX, cefotaxime; CAZ, ceftazidime; CPO, cefpirome; IPM, imipenem; MRP, meropenem; AK, amikacin; CIP, ciprofloxacin; TM/SMX, cotrimoxazole; TE, tetracycline; CS, colistin.

\begin{tabular}{|llllllllllllll|}
\hline & FOX & AMP & AUG & CTX & CAZ & CPO & IPM & MRP & AK & CIP & TM/SMX & TE & CS \\
\hline HA4 & $\leqslant 4(\mathrm{~S})$ & $8(\mathrm{~S})$ & $8(\mathrm{~S})$ & $\leqslant 1(\mathrm{~S})$ & $\leqslant 1(\mathrm{~S})$ & $\leqslant 1(\mathrm{~S})$ & $\leqslant 1(\mathrm{~S})$ & $\leqslant 0.25(\mathrm{~S})$ & $\leqslant 2(\mathrm{~S})$ & $\leqslant 0.25(\mathrm{~S})$ & $\leqslant 20(\mathrm{~S})$ & $\geqslant \mathbf{1 6}(\mathbf{R})$ & $\geqslant \mathbf{1 6}(\mathbf{R})$ \\
$\mathrm{HA} 112$ & $\leqslant 4(\mathrm{~S})$ & $8(\mathrm{~S})$ & $8(\mathrm{~S})$ & $\leqslant 1(\mathrm{~S})$ & $\leqslant 1(\mathrm{~S})$ & $\leqslant 1(\mathrm{~S})$ & $\leqslant 1(\mathrm{~S})$ & $\leqslant 0.25(\mathrm{~S})$ & $\leqslant 2(\mathrm{~S})$ & $\leqslant 0.25(\mathrm{~S})$ & $\leqslant 20(\mathrm{~S})$ & $4(\mathrm{~S})$ & $\geqslant \mathbf{1 6}(\mathbf{R})$ \\
HA14 & $\leqslant 4(\mathrm{~S})$ & $8(\mathrm{~S})$ & $8(\mathrm{~S})$ & $\leqslant 1(\mathrm{~S})$ & $\leqslant 1(\mathrm{~S})$ & $\leqslant 1(\mathrm{~S})$ & $\leqslant 1(\mathrm{~S})$ & $\leqslant 0.25(\mathrm{~S})$ & $\leqslant 2(\mathrm{~S})$ & $\leqslant 0.25(\mathrm{~S})$ & $\leqslant 20(\mathrm{~S})$ & $4(\mathrm{~S})$ & $\leqslant 0.5(\mathrm{~S})$
\end{tabular}

susceptible A. baumannii strains have been successfully treated (Bassetti et al., 2008) with intravenous colistin sulphomethate sodium plus rifampicin. Also, neither renal failure (among patients with normal baseline renal function) nor neurotoxicity were documented, so the role of colistin as a safe therapeutic option against difficultto-treat Gram-negative pathogens was emphasized (Bassetti et al., 2008).

Interestingly, none of the three patients studied had received colistin prior to the isolation of the polymyxin-resistant $H$. alvei strains. This was surprising, as crossresistance between colistin and antimicrobial compounds other than polymyxins has never been described, so previous exposure to carbapenems, ceftazidime, amikacin and ciprofloxacin (which all of the patients had received during hospitalization) could not explain the development of colistin resistance. One hypothesis is that previous administration of antibiotic compounds other than polymyxins may have altered membrane phospholipids, which led to lack of colistin activity due to irreversible modification of the bacterial target site. In fact, in vitro colistin resistance appeared to be a stable character, as it was documented even after thawing out and subculturing each strain many times. Anyway, this hypothesis is unlikely, given that colistin activity is the same as disinfectant activity (where no bacterial metabolism is required), so that alteration of cell membrane lipids (which is also a mechanism for so-called disinfectant resistance) is known to be reversible once the disinfectant is removed. Another possibility is that there was plasmid-mediated transfer of resistance genes, involving polymyxin resistance. The presence of mixed Gram-negative flora in the enteric environment may contribute to spread of resistance by DNA exchange. This has been described for diffusion of
ESBL genes, as well as for co-transferred aminoglycoside, fluoroquinolone, tetracycline and cotrimoxazole resistance, but never for reduced susceptibility to colistin (Savini et al., 2008). Both of these hypotheses then remain just speculative for the moment. Finally, it is likely that the two polymyxin-resistant $H$. alvei strains may have acquired resistance due to exposure of previously colonized patients to colistin. The two isolates could then have spread within the nosocomial environment and colonized two of the patients studied. The authors consider this hypothesis as the most plausible of the three mentioned. If this is what has really occurred, besides focusing on the first isolation of polymyxin-resistant $H$. alvei strains our findings further emphasize the need for the implementation of infection control measures to limit the nosocomial spread of uncommon organisms and the emergence of drug resistance among them.

\section{Acknowledgements}

We express our gratitude to Dr Claudio Cappetti (Liofilchem s.r.l., Roseto degli Abruzzi, Italy) and to Mrs Annarita Perfetti for their valuable assistance.

\section{Vincenzo Savini, ${ }^{1}$ Chiara Catavitello, ${ }^{1}$ Marzia Talia, ${ }^{1}$ Andrea Balbinot, ${ }^{1}$ Fabio Febbo, ${ }^{1}$ Arianna Pompilio, ${ }^{2}$ Giovanni Di Bonaventura, ${ }^{2}$ Raffaele Piccolomini ${ }^{2}$ and Domenico D'Antonio ${ }^{1}$}

\section{${ }^{1}$ Clinical Microbiology and Virology Unit, Department of Transfusion Medicine, 'Spirito Santo' Hospital, Pescara, Italy \\ ${ }^{2}$ Clinical Microbiology, Aging Research Center (Ce.S.I.), and Department of Biomedical Sciences, 'Gabriele d'Annunzio' University, Chieti, Italy}

Correspondence: Vincenzo Savini (vincsavi@yahoo.it)
Bassetti, M., Repetto, E., Righi, E., Boni, S., Diverio, M., Molinari, M. P., Mussap, M., Artioli, S., Ansaldi, F. \& other authors (2008). Colistin and rifampicin in the treatment of multidrug-resistant Acinetobacter baumannii infections. J Antimicrob Chemother 61, 417-420.

Catchpole, C. R., Andrews, J. M., Brenwald, N. \& Wise, R. (1997). A reassessment of the in-vitro activity of colistin sulphomethate sodium. $J$ Antimicrob Chemother 39, 255-260.

Gales, A. C., Reis, A. O. \& Jones, R. N. (2001). Contemporary assessment of antimicrobial susceptibility testing methods for polymyxin B and colistin: review of available interpretative criteria and quality control guidelines. J Clin Microbiol 39, 183-190.

Girlich, D., Naas, T., Bellais, S., Poirel, L., Karim, A. \& Nordmann, P. (2000). Biochemical-genetic characterization and regulation of expression of an ACC-1-like chromosome-borne cephalosporinase from Hafnia alvei. Antimicrob Agents Chemother 44, 1470-1478.

Janda, J. M. \& Abbott, S. L. (2006). The genus Hafnia: from soup to nuts. Clin Microbiol Rev 19, 12-18.

Jones, R. N., Anderegg, T. R. \& Swenson, J. M. the Quality Control Working Group (2005). Quality control guidelines for testing gramnegative control strains with polymyxin $B$ and colistin (polymyxin E) by standardized methods. J Clin Microbiol 43, 925-927.

Kasiakou, S. K., Michalopoulos, A., Soteriades, E. S., Samonis, G., Sermaides, G. J. \& Falagas, M. E. (2005). Combination therapy with intravenous colistin for management of infections due to multidrug-resistant gram-negative bacteria in patients without cystic fibrosis. Antimicrob Agents Chemother 49, 3136-3146.

Li, J., Nation, R. L., Milne, R. W., Turnidge, J. D. \& Coulthard, K. (2005). Evaluation of colistin as an agent against multi-resistant Gram-negative bacteria. Int J Antimicrob Agents 25, 11-25.

Markou, N., Apostolakos, H., Koumoudiou, C., Athanasiou, M., Koutsoukou, A., Alamanos, I. \& Gregorakos, L. (2003). Intravenous colistin in the treatment of sepsis from multiresistant Gram-negative bacilli in critically ill patients. Crit Care 7, R78-R83.

Muyembe, T., Vandepitte, J. \& Desmyter, J. (1973). Natural colistin resistance in Edwardsiella tarda. Antimicrob Agents Chemother 4, 521-524. 
NCCLS (2003). Performance Standards for Antimicrobial Disk Susceptibility Tests. Approved Standard M2-A8. Wayne, PA: National Committee for Clinical Laboratory Standards.

Savini, V., Catavitello, C., Talia, M., Di Berardino, F., Manna, A., Balbinot, A., Febbo, F. Carlino, D., Fioritoni, F. \& other authors (2008).
Ulcer infection by ESBL-producing Proteus mirabilis: a case report. Int J Low Extrem Wounds 7, 99-101.

Shimizu, S., lyobe, S. \& Mitsuhashi, S. (1977). Inducible high resistance to colistin in Proteus strains. Antimicrob Agents Chemother 12, $1-3$.
Tan, T. Y. \& Ng, S. Y. (2006). The in-vitro activity of colistin in gram-negative bacteria. Singapore Med J 47, 621.

Thomson, K. S., Sanders, C. C. \& Washington, J. A., II (1993). Ceftazidime resistance in Hafnia alvei. Antimicrob Agents Chemother 37, 13751376. 